\title{
Attracting pollinators vs escaping herbivores: eco-evolutionary dynamics of plants confronted with an ecological trade-off
}

\section{Authors}

Youssef Yacine ${ }^{1}$, Nicolas Loeuille ${ }^{1}$

1 Institute of Ecology and Environmental Sciences Paris (iEES Paris), Sorbonne Université/CNRS/IRD/INRA/Université de Paris/UPEC, 4 place Jussieu, 75252 Paris Cedex 5, France

\section{Corresponding author}

Youssef Yacine, youssef-yacine@hotmail.fr

Institute of Ecology and Environmental Sciences of Paris (iEES-Paris)

Tower 44-45, Floor 4, Room 413, case 237

4 place Jussieu, 75252 Paris Cedex 5, France

\section{Running headline}

Plant evolution: pollination vs. herbivory

\section{Statement of authorship}

N.L. and Y.Y. conceived the ideas. The analytical and numerical work was done by Y.Y. Results were analyzed by both N.L. and Y.Y. Y.Y. wrote the first draft of the manuscript that has been subsequently reviewed and edited by N.L. All authors contributed critically to the drafts and gave final approval for publication.

\section{Data accessibility statement}

No data were used in the present work. 


\begin{abstract}
A large number of plant traits are subject to an ecological trade-off between attracting pollinators and escaping herbivores. The interplay of both plant-animal interactions determines their evolution. Within a plant-pollinator-herbivore community in which interaction strengths depend on trait-matching, eco-evolutionary dynamics are studied using the framework of adaptive dynamics. We characterize the type of selection acting on the plant phenotype and the consequences for multispecies coexistence. We find that pollination favors stabilizing selection and coexistence. In contrast, herbivory fosters runaway selection, which threatens plant-animal coexistence. These contrasting dynamics highlight the key role of ecological trade-offs in structuring ecological communities. In particular, we show that disruptive selection is possible when such trade-offs are strong. While the interplay of pollination and herbivory is known to maintain plant polymorphism in several cases, our work suggests that it might also have fueled the diversification process itself.
\end{abstract}

\title{
Keywords
}

disruptive selection, pollination, herbivory, plant evolution, interaction pattern, stabilizing selection, runaway selection, multispecies coexistence 


\section{Introduction}

Flowering plants (i.e. angiosperms) are the most diverse and successful plant clade in terrestrial ecosystems, representing almost $90 \%$ of the species described (HernándezHernández \& Wiens 2020). Most of them rely on animals for pollination (Ollerton et al. 2011). Because animal pollination can favor reproductive isolation, it has early been proposed as an important diversity driver among angiosperms (Grant 1949). Plant-herbivore interactions have, however, also been identified as potentially fostering diversification due to the ensuing evolutionary arms race between interacting antagonists (Ehrlich \& Raven 1964). These two non-mutually exclusive hypotheses are supported by several phylogenetic investigations. Plantpollinator coevolution explains higher diversification rates within angiosperms (Hodges \& Arnold 1995; Sargent 2004; Hernández-Hernández \& Wiens 2020), but plant-herbivore coevolution also spurs plant diversification as a result of defense-counterdefense innovations (Farrell et al. 1991; Becerra et al. 2009). Plant phylogenies also reveal that plant-pollinator and plant-herbivore coevolution are very often inextricably intertwined (e.g. Armbruster 1997), which advocates for an integrative framework accounting for both interaction types (Fontaine et al. 2011).

A large number of plant traits are notably under conflicting selection due to the interplay between pollination and herbivory (Strauss \& Irwin 2004), floral traits in particular (Strauss \& Whittall 2006). Conflicting selection pressures can emerge from several mechanisms acting either in isolation or synergistically. Herbivory-induced changes in plant chemistry potentially reduce pollinator visitations (e.g. Kessler et al. 2011). Many plant traits acting on the plant visibility (size, phenology, floral display, volatile compounds) may increase herbivory (apparency hypothesis, Feeny 1976) while also attracting allies (e.g. Brody 2008). Genetic correlation can also exist between two plant traits, each involved in one plant-animal interaction 
(Strauss et al. 2004). A decisive consequence is that the selection pressures in the absence of either one animal species would be modified in the presence of both animal groups (Ramos \& Schiestl 2019). Conflicting selection is very often due to shared preferences for plant phenotypes between pollinators and herbivores, a pattern that is largely widespread in nature (Strauss \& Whittall 2006) and that promotes the stable coexistence of the community (Yacine \& Loeuille 2021). This preference pattern indicates that plant species are subject to an ecological trade-off between attracting pollinators and escaping herbivores (Strauss et al. 2002). This trade-off has interestingly been shown to support the maintenance of a flower-color polymorphism in the wild radish Raphanus sativus (Irwin et al. 2003), which suggests that the interplay between pollination and herbivory could also lead to disruptive selection and promote diversification.

Conflicting selection arises because pollination and herbivory exert opposite pressures on plant traits (Thompson 2009), with contrasting implications in terms of diversification potential (Yoder \& Nuismer 2010).

As stronger mutualism increases fitness, plants are selected to better match their pollinator phenotype and vice versa (e.g. spur length and pollinator tongue, Whittall \& Hodges 2007) so that stabilizing selection is expected (e.g. Kiester et al. 1984). Pollination-induced stabilizing selection has been empirically characterized several times (e.g. Parachnowitsch \& Kessler 2010). It disfavors extreme phenotypes, thus reducing the potential for disruptive selection (Kopp \& Gavrilets 2006; Yoder \& Nuismer 2010; Maliet et al. 2020). Note, however, that this does not imply that mutualism cannot promote diversification, but rather that additional processes are required, many of which are presented in the review by Chomicki et al. (2019). For instance, as a result of increased densities, mutualism can exacerbate intraspecific competition, which in turn favors disruptive selection (Bolnick 2004; Yoder \& Nuismer 2010). 
Conversely, predation reduces prey fitness so that plants are selected to escape herbivore preferences (e.g. chemical defenses and herbivore tolerance, Becerra et al. 2009). Such arms races dynamics (Ehrlich \& Raven 1964; Dawkins \& Krebs 1979) expose the plant species to runaway selection, as observed in empirical systems (e.g. Mauricio \& Rausher 1997). Because apparent competition (Holt 1977) imposes a cost on phenotype matching between conspecific plants, herbivory also favors increased plant phenotypic divergence thereby enhancing disruptive selection (Kopp \& Gavrilets 2006; Yoder \& Nuismer 2010; Maliet et al. 2020).

The ecological implications of such conflicting selection are far-reaching. The relative interaction strength of pollination vs. herbivory notably constrains coexistence within plantpollinator-herbivore communities (Mougi \& Kondoh 2014; Sauve et al. 2016a; Yacine \& Loeuille 2021). A balance between the two interactions is expected to foster coexistence. Because interaction strengths are the result of plant-animal coevolution, the evolution of plant traits under conflicting selection has potentially important consequences for multispecies coexistence.

In the present paper, our goals are (1) to determine how the interplay between pollination and herbivory drives the evolution of plant traits involved in both interactions, (2) to understand the consequences in terms of interaction patterns and multispecies coexistence, (3) to assess how ecological trade-offs may lead to stabilizing vs. disruptive selection. We model a community - a plant species interacting simultaneously with a pollinator and a herbivore species - in which plant-animal interactions depend on a single plant trait involved in both interactions. Each interaction increases in strength with the matching of plant and animal phenotypes. Examples of such traits include flower color (Irwin et al. 2003), flower display (Galen \& Cuba 2001), chemical volatiles (Theis et al. 2014) and the corresponding animal preferences or detection abilities. Phenological matching (e.g. Brody 2008) would also fit our 
framework well. Using the framework of adaptive dynamics (Metz et al. 1992; Dieckmann \& Law 1996), we determine how evolutionary dynamics depend on pollination and herbivory.

In particular, we modulate the strength of the trade-off by manipulating the dissimilarity between animal phenotypes (x-axis in Fig. 1A). Under weak ecological trade-offs (i.e. dissimilar animal preferences, Fig. 1A-right), we expect selection to favor plant phenotypes away from the herbivore phenotype. Such runaway dynamics may simultaneously lead away from the pollinator phenotype, resulting in the loss of both animals (Fig. 1B.1a). Conversely, these dynamics can converge towards the pollinator phenotype around which they eventually stabilize before herbivores are lost (Fig. 1B.2). If herbivores are lost, stabilizing selection provided by pollinators eventually prevails (temporality, Fig. 1B.1b). In general, because interactions are unbalanced under weak trade-offs, we expect coexistence to be rarely favored. Under strong ecological trade-offs (i.e. similar animal preferences, Fig. 1A-left), interactions are intrinsically correlated, so that coexistence should be more systematically observed. As herbivory and pollination selection effects are more balanced, selection regimes may vary from stabilizing to disruptive depending on parameter scenarios. In the latter case, stable plant dimorphism may emerge (Fig. 1B.3). 


\section{A Expected type of selection} \& ecological outcome

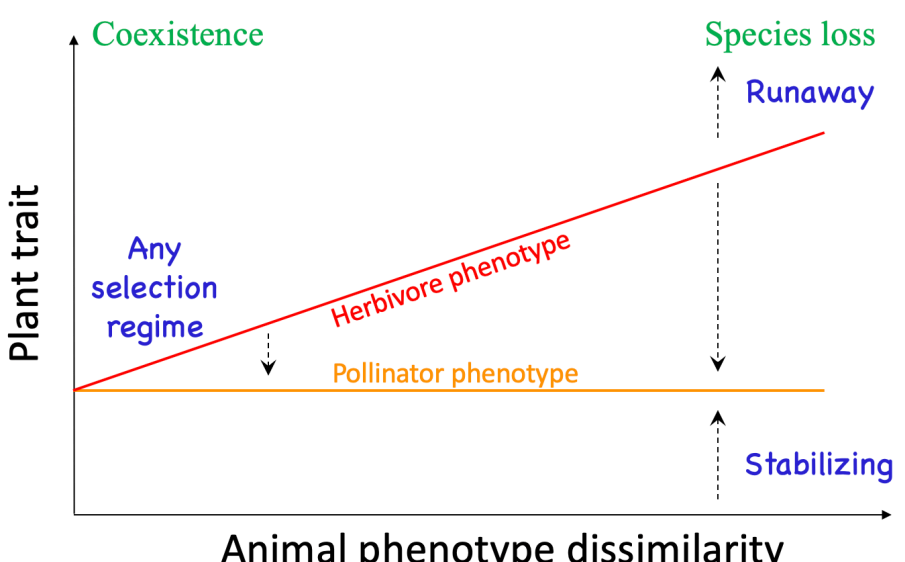
ecological Weak trade-off ecological trade-off

\section{B Resulting ecological community}

\section{Runaway selection}

a.<smiles>CC(C)(C)C(C)(C)C</smiles>

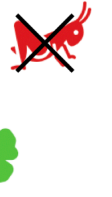

2. Stabilizing selection

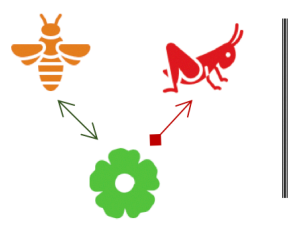

3. Disruptive selection

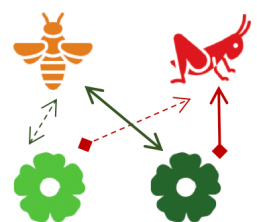

Fig. 1: Expected eco-evolutionary dynamics within the plant-pollinator-herbivore community. A. The type of selection and the ecological outcome depend on the dissimilarity between animal phenotypes (i.e. preferences), which is a proxy for the strength of the ecological trade-off. B. The long-term community composition depends on the type of selection. (1) Runaway selection leads to the extinction of a first animal species as the plant phenotype is diverging. (a) Pollinators are lost first so that runway selection continues until herbivores are also lost. (b) Herbivores are lost first so that selection turns stabilizing (i.e. temporality), leading to a perfect plantpollinator matching. (2) Stabilizing selection can enable the maintenance of coexistence. (3) Disruptive selection leads to the emergence of plant dimorphism.

\section{Model \& Methods}

\section{Model}

\section{Population dynamics}

We model the dynamics of a plant-pollinator-herbivore module $(P, M, H$ respectively) using a Lotka-Volterra framework: 


$$
\begin{gathered}
\frac{d P}{d t}=P\left(r_{p}-c_{p} P+a_{p m} M-a_{p h} H\right) \\
\frac{d M}{d t}=M\left(r_{m}-c_{m} M+e_{m} a_{p m} P\right) \\
\frac{d H}{d t}=H\left(r_{h}-c_{h} H+e_{h} a_{p h} P\right)
\end{gathered}
$$

Plants are assumed to have a positive intrinsic growth rate $\left(r_{p}>0\right.$, autotrophs), while both pollinator $\left(r_{m}<0\right)$ and herbivore growth rates $\left(r_{h}<0\right)$ are assumed negative (heterotrophs). Plants $\left(c_{p}\right)$, pollinators $\left(c_{m}\right)$, and herbivores $\left(c_{h}\right)$ undergo intraspecific competition. The strength of interspecific interactions is given by $a_{p m}$ for pollination and $a_{p h}$ for herbivory. $e_{m}$ and $e_{h}$ are the conversion efficiencies from plants to animals. See table $\mathbf{1}$ for details.

Ecological dynamics associated with system (1) lead to stable coexistence when the strengths of pollination and herbivory are balanced (Yacine \& Loeuille 2021). The analytical expression of the coexistence equilibrium $\left(P^{*}, M^{*}, H^{*}\right)$ is given in Appendix A.I. Here, we want to investigate how plant evolution constrains the interaction balance (i.e. pollination vs. herbivory) and thereby coexistence.

\section{Plant-animal interactions depend on trait-matching}

We assume plant-animal interactions to intensify as the matching between plant $\left(t_{p}\right)$ and animal phenotypes - pollinator $\left(t_{m}\right)$ or herbivore $\left(t_{h}\right)$ - increases (equations (2)). Consider, for instance, the color of plant flowers $\left(t_{p}\right)$ that is preferred $\left(t_{m}, t_{h}\right)$ by animal species, expressed in wavelength to be quantitative (e.g. Irwin et al. 2003). Consider also phenological traits (e.g. Brody 2008) such as the date of flowering $\left(t_{p}\right)$ in relationship with the timing of maximal seasonal activity $\left(t_{m}, t_{h}\right)$. 


\begin{tabular}{|c|c|}
\hline$a_{p m}\left(t_{p}\right)=\frac{a_{p m 0}}{\sqrt{2 \pi \sigma_{\text {Pol }}^{2}}} \exp \left[-\frac{\left(t_{p}-t_{m}\right)^{2}}{2 \sigma_{\text {Pol }}^{2}}\right]$ & (2) \\
\cline { 1 - 2 }$a_{p h}\left(t_{p}\right)=\frac{a_{p h 0}}{\sqrt{2 \pi \sigma_{\text {Her }}^{2}}} \exp \left[-\frac{\left(t_{p}-t_{h}\right)^{2}}{2 \sigma_{\text {Her }}^{2}}\right]$ & \\
\hline
\end{tabular}

As $\sigma_{P o l}$ controls how quickly the strength of pollination decreases with plant-pollinator phenotype dissimilarity, it corresponds to the pollination-niche width, which depends on the generalism of both species. Likewise, $\sigma_{H e r}$ embodies the herbivory-niche width. $a_{p m 0}$ and $a_{p h 0}$ correspond to basal interaction rates. See table 1 for details.

Table 1: List of all model parameters and variables with their biological significance, value and dimension ( $\boldsymbol{M}$ for mass, $\boldsymbol{L}$ for length and $\boldsymbol{t}$ for time).

\begin{tabular}{|c|c|c|c|c|}
\hline \multicolumn{2}{|c|}{$\begin{array}{c}\text { Variables and } \\
\text { parameters }\end{array}$} & \multirow{2}{*}{$\begin{array}{c}\text { Biological } \\
\text { significance } \\
\text { Plant biomass density }\end{array}$} & \multirow[t]{2}{*}{ Value } & \multirow{2}{*}{$\begin{array}{c}\text { Dimension } \\
M \cdot L^{-2}\end{array}$} \\
\hline \multirow{4}{*}{ Variables } & $P$ & & & \\
\hline & $M$ & $\begin{array}{c}\text { Pollinator biomass } \\
\text { density }\end{array}$ & & $M \cdot L^{-2}$ \\
\hline & $H$ & $\begin{array}{c}\text { Herbivore biomass } \\
\text { density }\end{array}$ & & $M \cdot L^{-2}$ \\
\hline & $t_{p}$ & Plant phenotype & & Dimensionless \\
\hline \multirow{7}{*}{$\begin{array}{c}\text { Interspecific } \\
\text { interaction } \\
\text { parameters }\end{array}$} & $a_{p m 0}$ & $\begin{array}{l}\text { Basal pollination rate } \\
\text { (per capita) }\end{array}$ & {$[3,9]$} & $\left(M \cdot L^{-2}\right)^{-1} \cdot t^{-1}$ \\
\hline & $a_{p h 0}$ & $\begin{array}{l}\text { Basal herbivory rate } \\
\text { (per capita) }\end{array}$ & {$[3,9]$} & $\left(M \cdot L^{-2}\right)^{-1} \cdot t^{-1}$ \\
\hline & $t_{m}$ & Pollinator phenotype & 0 & Dimensionless \\
\hline & $t_{h}$ & Herbivore phenotype & {$[0,3]$} & Dimensionless \\
\hline & $t_{h}-t_{m}$ & $\begin{array}{l}\text { Strength of ecological } \\
\text { trade-off }\end{array}$ & {$[0,3]$} & Dimensionless \\
\hline & $\sigma_{P o l}$ & Pollination niche width & {$[1.5,3]$} & Dimensionless \\
\hline & $\sigma_{\mathrm{Her}}$ & Herbivory niche width & {$[1.5,3]$} & Dimensionless \\
\hline \multirow{2}{*}{$\begin{array}{c}\text { Other } \\
\text { ecological } \\
\text { parameters }\end{array}$} & $r_{p}$ & $\begin{array}{l}\text { Plant intrinsic growth } \\
\text { rate }\end{array}$ & 10 & $t^{-1}$ \\
\hline & $r_{m}$ & $\begin{array}{c}\text { Pollinator intrinsic } \\
\text { growth rate }\end{array}$ & -1 & $t^{-1}$ \\
\hline
\end{tabular}




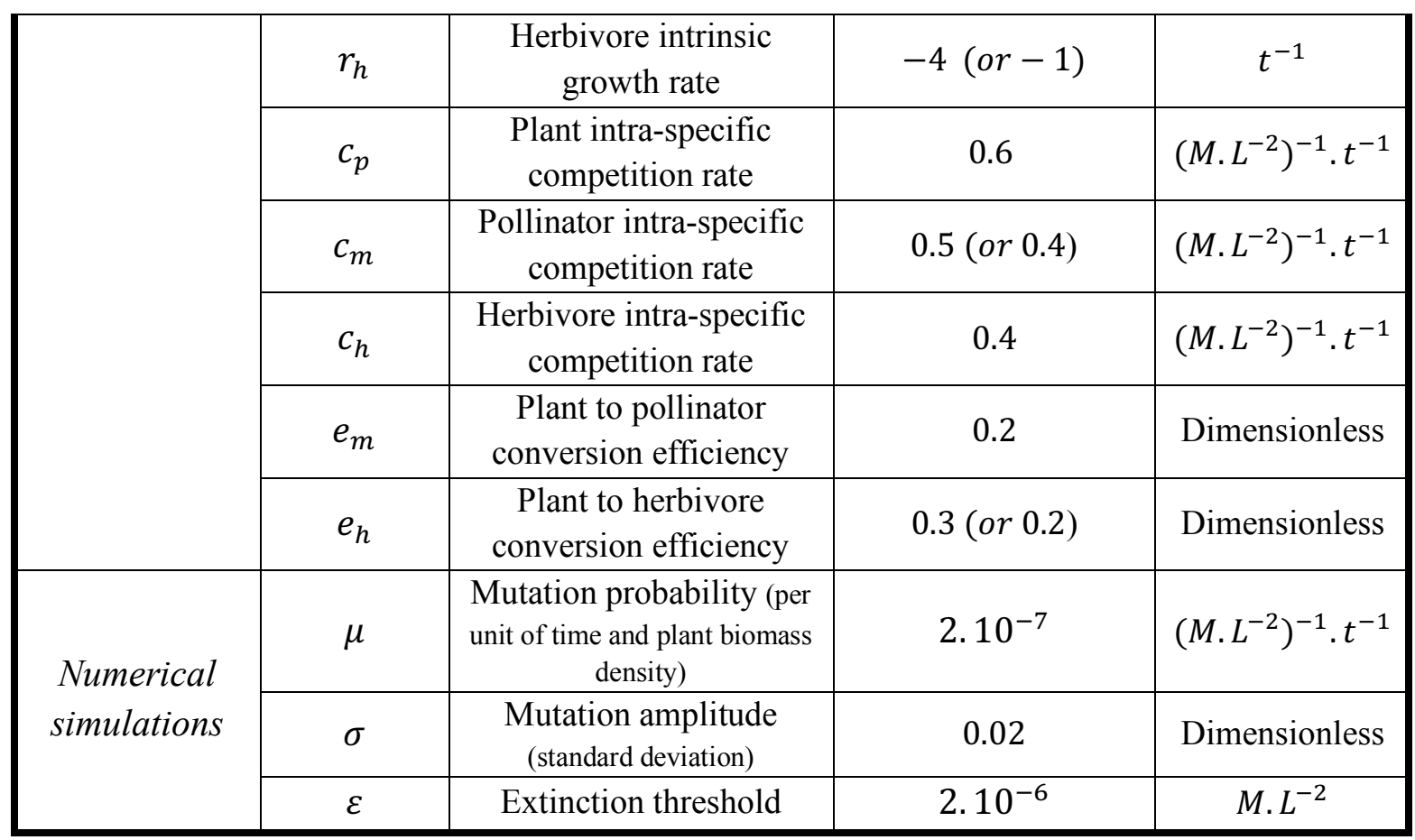

\section{Methods}

Animal phenotypes are fixed parameters (without loss of generality: $t_{m}=0, t_{h} \geq t_{m}$ ) while we study the evolution of the plant phenotype $\left(t_{p}\right)$. Using the framework of adaptive dynamics, we characterize the type of selection acting on the plant phenotype, as well as its consequences on coexistence (see below). Parameters directly affecting plant-animal interactions - the interspecific parameter set $\left(t_{h}-t_{m}, a_{p m 0}, a_{p h 0}, \sigma_{P o l}, \sigma_{H e r}\right)-$ are at the core of our investigation. In particular, the dissimilarity between animal phenotypes $\left(t_{h}-t_{m}\right)$ constrains the strength of the ecological trade-off affecting plant evolution (Fig. 1A, detailed in Appendix B.I). When animals are very similar $\left(t_{m} \approx t_{h}\right)$, any plant evolution toward more pollinator attraction directly increases herbivory, and any escape from herbivory comes with a direct cost in pollination (strong trade-off). An important dissimilarity (i.e. $t_{m} \ll t_{h}$ ) allows plants to escape herbivory while paying little cost in terms of pollination (weak trade-off). 
Thanks to the model simplicity, several analytical results uncovering various aspects of evolutionary dynamics (e.g. equation (3)) are possible. For aspects that cannot be mathematically investigated, we provide numerical resolutions characterizing the variation of eco-evolutionary dynamics along the gradient (x-axis in Fig. 1A) of trade-off intensity (E3diagrams, e.g. Fig. 2a). To broaden our understanding of possible evolutionary dynamics, we undertake Monte Carlo experiments (details in appendix B.III). We let interspecific parameters vary independently within their interval ranges (table 1), which were chosen to explore a wide range of pollination and herbivory intensities (Appendix B.II). Remaining parameters $\left(r_{p}, r_{m}, r_{h}, c_{p}, c_{m}, c_{h}, e_{m}, e_{h}\right)$ are fixed, but the experiments were conducted for two different sets to assess their robustness. The different Monte Carlo experiments are presented directly in the Result section when needed. Our approach is finally complemented by numerical simulations of the community eco-evolutionary dynamics. Such simulations are used to investigate the emergence of plant dimorphism in the case of disruptive selection.

\section{Adaptive dynamics and type of selection}

Within a monomorphic plant population with phenotype $t_{p}$ (resident), we investigate whether a mutant with a new phenotype $t_{p}{ }^{\prime}$ can invade. Invasion fitness $w\left(t_{p}{ }^{\prime}, t_{p}\right)$ can then be computed as the per capita growth rate of that mutant, when rare and in the environment $\left(P^{*}, M^{*}, H^{*}\right)$ set by the resident population (Metz et al. 1992, see appendix A.II.1). When a mutant invades (i.e. $w\left(t_{p}{ }^{\prime}, t_{p}\right)>0$ ), it replaces the resident population, thus becoming the new resident. The consecutive sequences of trait substitution define the long-term evolutionary dynamics. Assuming small and rare mutational steps, the variation of phenotype $t_{p}$ is proportional to the selection gradient (equation (3)), i.e. the derivative of invasion fitness with respect to the mutant's trait (Dieckmann \& Law 1996). Evolutionary singularities $t_{p}^{*}$ correspond to phenotypes that nullify the selection gradient. 
The type of selection - stabilizing, disruptive, or runaway - acting on the plant trait. depends on (1) the properties of the evolutionary singularities, and (2) the position of the plant phenotype relative to these singularities. Two independent properties - convergence and invasibility - characterize evolutionary singularities (criteria in appendix A.II.1). Convergence indicates whether the trait evolves toward the singularity in its vicinity. Two types of singularities are convergent - the non-invasible continuously stable strategy (CSS, Eshel, 1983) and the invasible branching point (Geritz et al. 1997). Invasibility specifies whether the singularity may be invaded by nearby mutants (i.e. is an ESS, Maynard Smith \& Price 1973). In a CSS vicinity, evolution leads to the CSS phenotype which is maintained by stabilizing selection (e.g. Fig. 2b). Selection is thus stabilizing in the basin of attraction of a CSS. Plantpollinator-herbivore coexistence is notably maintained if a CSS is reached while the three species coexist (Fig. 1B.2). In contrast, a branching point yields the emergence of a stable plant dimorphism (Fig. 1B.3) due to disruptive selection (e.g. Fig. 2c). Accordingly, selection is disruptive in the basin of attraction of a branching point. Finally, phenotypes that are not in the basin of attraction of a convergent singularity are under runaway selection. This is possible in the presence of a non-convergent singularity, i.e. a repellor. Note that runaway selection always leads to the disruption of plant-pollinator-herbivore coexistence (Fig. 1B.1, e.g. Fig. 2c). We illustrate how the proportion of phenotypic space under each type of selection is calculated in Appendix B.III.1.

This framework is completed by numerical simulations starting from a monomorphic plant population $\left(t_{p}\right)$ interacting with a pollinator $\left(t_{m}\right)$ and a herbivore $\left(t_{h}\right)$ population. Mutations are randomly generated following a Poisson process characterized by a mutation probability per unit of time and plant biomass density $\mu=2 \cdot 10^{-7}$. Proportionally to phenotype abundances, a parent phenotype is randomly chosen at each mutation event. The mutant phenotype is drawn from a Gaussian distribution centered around the parent phenotype with 
standard deviation $\sigma=0.02$. Its initial density is set to a small value $\varepsilon$ which also corresponds to the extinction threshold, taken from the parent population.

\section{Results}

The key role of the ecological trade-off

Eco-evolutionary dynamics qualitatively differ depending on the strength of the ecological trade-off (Fig. 2). Selection is either stabilizing or runaway under weak trade-offs, while disruptive selection is only possible when the trade-off is strong or intermediate.

At strong or intermediate trade-offs $\left(t_{h}-t_{m} \leq 2\right.$ in Fig. 2a), stabilizing selection allows coexistence. Depending on initial plant phenotype, such an outcome arises either directly through the evolution toward a CSS (e.g. starting point b in Fig. 2a, Fig. 2b), or after transitory branching dynamics (e.g. starting point c in Fig. 2a, Fig. 2c). In the latter case, disruptive selection at branching favors both upper (weaker plant-animal interactions) and lower (stronger interactions) plant phenotypes, leading to the coexistence of two increasingly dissimilar phenotypes (Fig. 2c). The upper branch eventually goes extinct due to competition between morphs, while the lower branch converges toward the CSS phenotype (Fig. 2c). In this example, the fitness valley arising from the presence of herbivores - i.e. the repellor separating the branching and CSS basins of attraction (grey curve in Fig. 2a) - can be crossed thanks to the emergence of dimorphism.

Coexistence is often disrupted by runaway selection when the trade-off gets weaker $\left(t_{h}-t_{m}>2\right.$ in Fig. 2a). A new repellor then appears. Starting above the repellor (e.g. point d in Fig. 2a, Fig. 2d), plant-animal interactions become increasingly weak, which leads to the extinction of pollinators, then of herbivores (Fig. 2d). Coexistence is maintained when the plant phenotype is initially in the CSS basin of attraction (e.g. point e in Fig. 2a). Interestingly, this 
coexistence leads to very unbalanced interactions (strong pollination, weak herbivory). When the trade-off gets even weaker (e.g. point $\mathrm{f}$ in Fig. 2a), the CSS eventually leaves the coexistence area. Runaway selection continuously decreases herbivory eventually leading to the extinction of herbivores and to a perfectly matching plant-pollinator community.
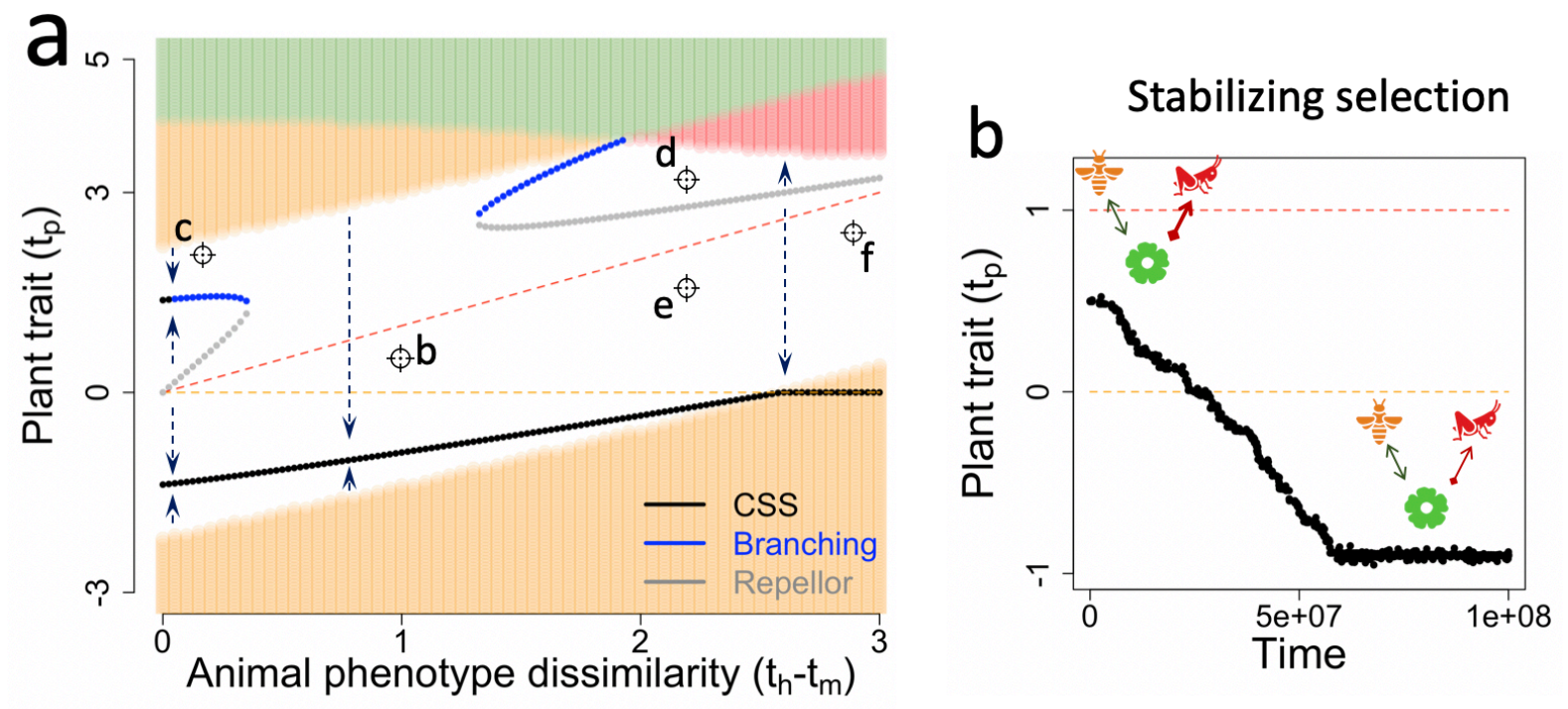

Community $\square$ plants $\square$ plants \& pollinators \& herbivores
composition $\square$ plants \& pollinators $\square$ plants \& herbivores
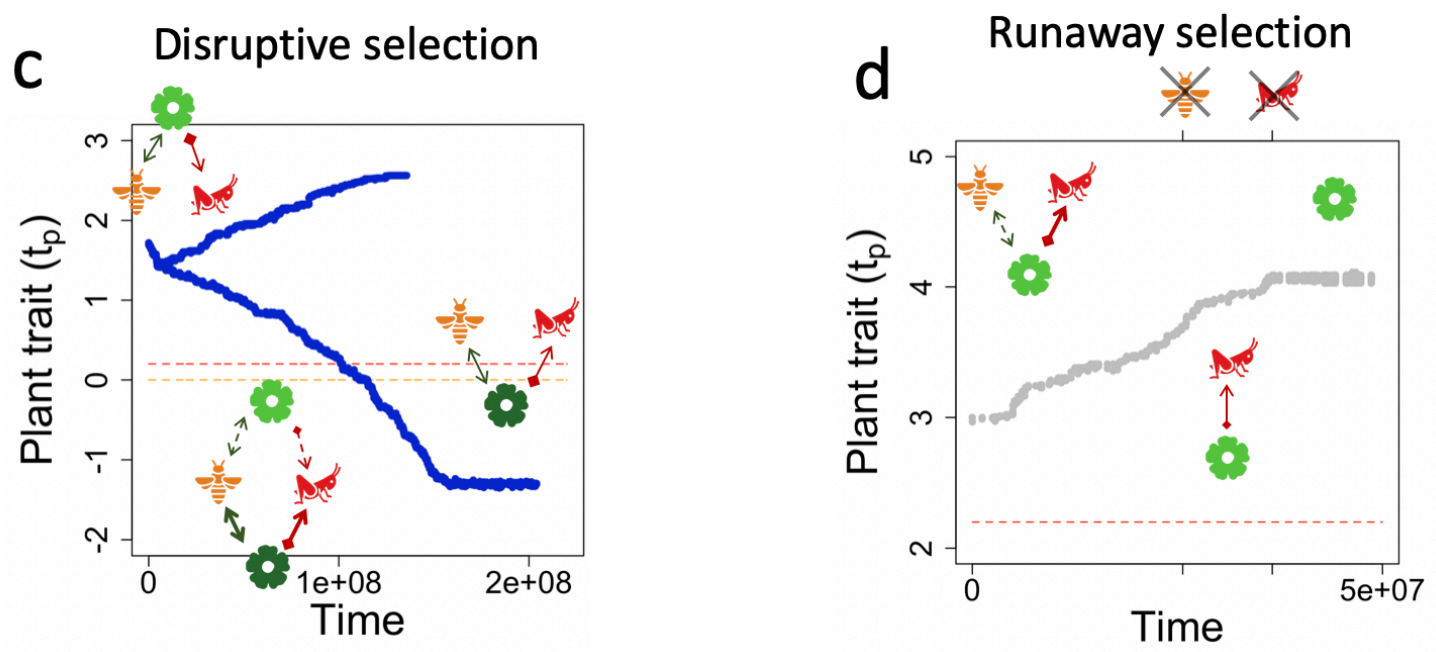

Fig. 2: Qualitative variations of community eco-evolutionary dynamics with trade-off intensity. Parameter set: ecological $\left(r_{p}=10, r_{m}=-1, r_{h}=-4, c_{p}=0.6, c_{m}=0.5, c_{h}=0.4, e_{m}=0.2, e_{h}=0.3\right)$; interspecific $\left(t_{m}=0, a_{p m 0}=5, a_{p h 0}=7, \sigma_{P o l}=3, \sigma_{H e r}=2.8\right)$. a. Eco-evolutionary dynamics change with the trade-off intensity. Arrows indicate the direction of evolutionary trajectories, and the background colors indicate the community composition. b. Simulated evolutionary dynamics under stabilizing selection. The initial plant 
phenotype corresponds to point $\mathrm{b}$ in $2 . \mathrm{a}\left(t_{h}=1\right.$ i.e. intermediate trade-off). c. Simulated evolutionary dynamics under disruptive selection. The initial plant phenotype corresponds to point $\mathrm{c}$ in 2 . $\mathrm{a}\left(t_{h}=0.2\right.$ i.e. strong tradeoff). d. Simulated evolutionary dynamics under runaway selection. The initial plant phenotype corresponds to point $d$ in 2 .a $\left(t_{h}=2.2\right.$ i.e. weak trade-off). Small pictograms depict the community composition, with arrow thickness capturing interaction strengths.

\section{The opposite effect of pollination vs. herbivory}

The direction of plant phenotype evolution is given by the selection gradient (equation (3)). It shows that the plant phenotype is under two contrasting selective forces (i.e. opposite sign). For a plant phenotype outside $\left[t_{m}, t_{h}\right]$, pollination selects for phenotypes that are closer to animal phenotypes, while herbivory selects for phenotypes that are further away from animal phenotypes. Pollination and herbivory are therefore a converging and a diverging selective force, respectively.

$$
\frac{d t_{p}}{d t} \propto \underset{\text { gradient }}{\text { Selection }}=-\underbrace{a_{p m}\left(t_{p}\right) M^{*}}_{\text {pollination }} \frac{\left(t_{p}-t_{m}\right)}{\sigma_{\text {Pol }}^{2}}+\underbrace{a_{p h}\left(t_{p}\right) H^{*}}_{\text {herbivory }} \frac{\left(t_{p}-t_{h}\right)}{\sigma_{H e r}^{2}}
$$

Considering an evolutionary singularity $t_{p}^{*}$, it is also possible to demonstrate that pollination and herbivory have opposite effects on its evolutionary stability (i.e. non-invasibility). Singularities are invasible if inequality (4) is satisfied (proof and expression of $f\left(t_{p}^{*}\right)$ in Appendix A.II.2). Evolutionary stability is thus favored by pollination and disfavored by herbivory.

$$
\text { Invasibility } \Leftrightarrow-\underbrace{a_{\text {pm }}\left(t_{p}^{*}\right) M^{*}}_{\text {pollination }}\left[1+\frac{f\left(t_{p}^{*}\right)^{2}}{\sigma_{\text {Pol }}^{2}}\right]+\underbrace{a_{p h}\left(t_{p}^{*}\right) H^{*}}_{\text {herbivory }}\left[1+\frac{f\left(t_{p}^{*}\right)^{2}}{\sigma_{\text {Her }}^{2}}\right]>0
$$

To assess how the type of selection depends on pollination vs. herbivory, we rely on our first Monte Carlo experiment (MC1) as the convergence of evolutionary singularities is not analytically tractable. We sampled 10000 interspecific parameter sets and determined the position and nature of all evolutionary singularities allowing ecological coexistence, as well as 
interaction strengths and animal densities at these singularities. The resulting dataset thereby links the ratio of pollination to herbivory at the singularity $\left.\left(\frac{a_{p m} M^{*}}{a_{p h} H^{*}}\right]_{t_{p}^{*}}\right)$ to the type of selection (Fig. 3a). Stabilizing, runaway and disruptive selections are characterized by contrasting pollination to herbivory ratios. The ratio explains around two-thirds of the variance in selection type (Kruskal-Wallis, $p_{\text {value }}<2 \cdot 10^{-16}$ ). High pollination is largely associated with stabilizing selection, high herbivory with runaway selection, while disruptive selection happens for intermediate situations (Fig. 3a). These results notably imply that the convergence of evolutionary singularities (i.e. convergence stability, Eshel 1983) is favored by pollination and disfavored by herbivory. Disruptive selection requires a balance between pollination and herbivory because strong herbivory relative to pollination fosters invasibility (inequality (4)), but convergence is lost when herbivory is too strong, leading to runaway dynamics (Fig. 3a). These key results derived from our Monte Carlo experiment are robust to the modification of the ecological parameter set (Appendix C.II, Fig. S4a). Note finally that "simpler" ratios - the ratios of interaction strengths $\left.\left(\frac{a_{p m}}{a_{p h}}\right]_{t_{p}^{*}}\right)$ or animal densities $\left.\left(\frac{M^{*}}{H^{*}}\right]_{t_{p}^{*}}\right)$ - have also a large and significant power in explaining the nature of selection (Appendix C.I).

The structuring role of the ecological trade-off can be clarified using the relationship between the relative strength of pollination vs. herbivory and the type of selection.

Firstly, selection is either stabilizing or runaway at weak trade-offs while all types of selection, including disruptive, are possible at strong or intermediate trade-offs (Fig. 3b, e.g. Fig. 2a). This result, which is robust to the modification of the ecological parameter set (Appendix C.II, Fig. S4b), is obtained from our second Monte Carlo experiment (MC2). We divided the potential range of trade-off intensities (i.e. $t_{h}-t_{m}$, table 1) into six equal intervals to sample from, but without constraining the sampling of the other interspecific parameters. For 
each sampling (6 x 1000), we calculated the proportion of phenotypic space associated with each type of selection (Appendix B.III.1), thereby estimating the effect of trade-off intensity on selection (Fig. 3b). Disruptive selection is only possible at strong or intermediate trade-offs (Fig. 3b, e.g. Fig. 2a) because it requires a balance between pollination and herbivory (Fig. 3a). Balanced ratios are possible when the plant phenotype is approximately equidistant from animal phenotypes, which is the case when the trade-off is strong as animal phenotypes are similar. When animal phenotypes are dissimilar, the selective gradient (equation (3)) is strictly negative in between animal phenotypes (i.e. in $\left[t_{m}, t_{h}\right]$, selection increases plant-pollinator matching while reducing plant-herbivore matching). No singularity is possible, branching in particular. Rather than phenotypic equidistance, balanced ratios may be obtained when pollination and herbivory are both weak, owing to narrow interaction niche widths for instance. This latter case explains the occurrence of disruptive selection at intermediate trade-offs corresponding to dissimilar animal phenotypes. When animals have very dissimilar phenotypes, pollination to herbivory ratios are either large or small outside $\left[t_{m}, t_{h}\right]$, depending on which animal phenotype is closer to the plant phenotype. As a result, selection is either stabilizing or runaway when the ecological trade-off is weak (Fig. 3b, e.g. Fig. 2a).

Secondly, when coexistence is maintained, the long-term interaction pattern is balanced at strong trade-offs but becomes increasingly biased toward stronger pollination than herbivory as the trade-off weakens (Fig. 3c). This result, which is robust to the modification of the ecological parameter set (Appendix C.II, Fig. S4c), derives from our third Monte Carlo experiment (MC3). Using the same procedure as MC2 (6 x 1000 samplings), we assessed how trade-off intensity constrains $\left(t_{h}-t_{m}\right)$ the contribution of pollination to total plant-animal interactions at the evolutionary endpoints. Such long-term dynamics correspond either to CSS phenotypes under stabilizing selection (Fig. 1B.2), or to post-branching communities with 
maintained dimorphism (Fig. 1B.3). Simulating community eco-evolutionary dynamics, we find that dimorphism is maintained following approximately $5 \%$ of the branching events encountered and only for very strong trade-offs (Appendix D.III, e.g. Fig. S7). In most cases, disruptive selection eventually leads to the loss of one of the two phenotypes, ending at a CSS at which stabilizing selection prevails (e.g. Fig. 2c). As a result, it is stabilizing selection which almost always supports the maintenance of coexistence (more than $99 \%$ of the cases encountered in MC3, Appendix B.III.4). As pollination provides stabilizing selection (Fig. 3a), the long-term interaction pattern is biased toward stronger pollination than herbivory. As the trade-off weakens, this increasing bias (Fig. 3c) implies that coexistence gets increasingly fragile. Herbivores are indeed likely closer to extinction owing to a weak plant-herbivore interaction, while the stability of the ecological system is threatened (Yacine \& Loeuille 2021). For weak trade-offs, runway selection also gets more frequent eventually destroying coexistence (Fig. 3b, but not observed with our second ecological parameter set, Fig. S4b in appendix C.II). 
a.

\section{Nature of selection \& Ratio of pollination to herbivory}

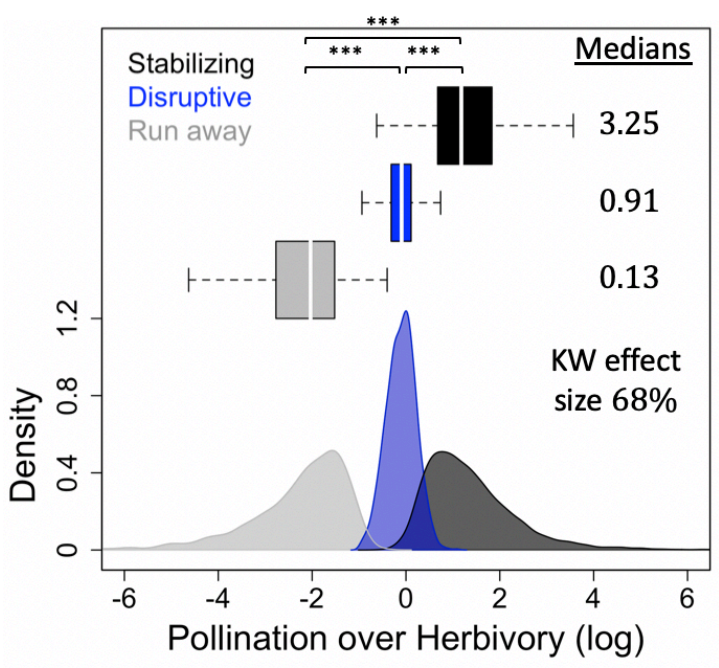

$$
\left.\frac{a_{p m} M^{*}}{a_{p h} H^{*}}\right]_{t_{p}^{*}}
$$

\section{b. Selection depends on trade-off intensity}

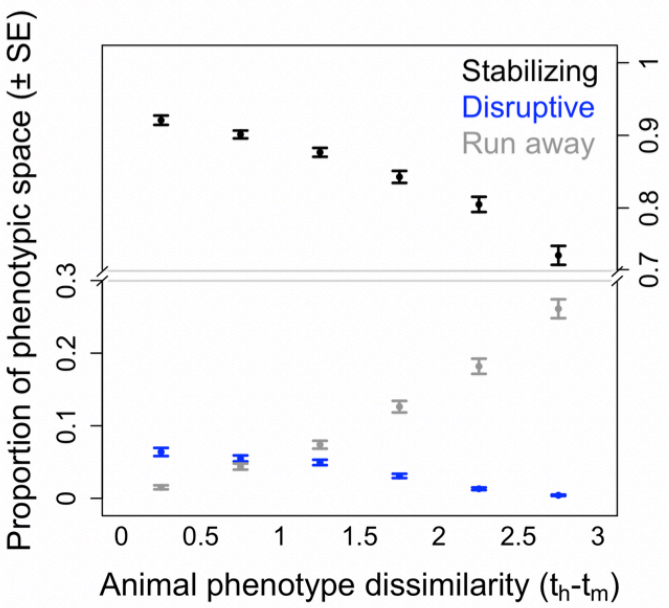

\section{Strong}

trade-off

Weak

trade-off

\section{Long-term interaction pattern}

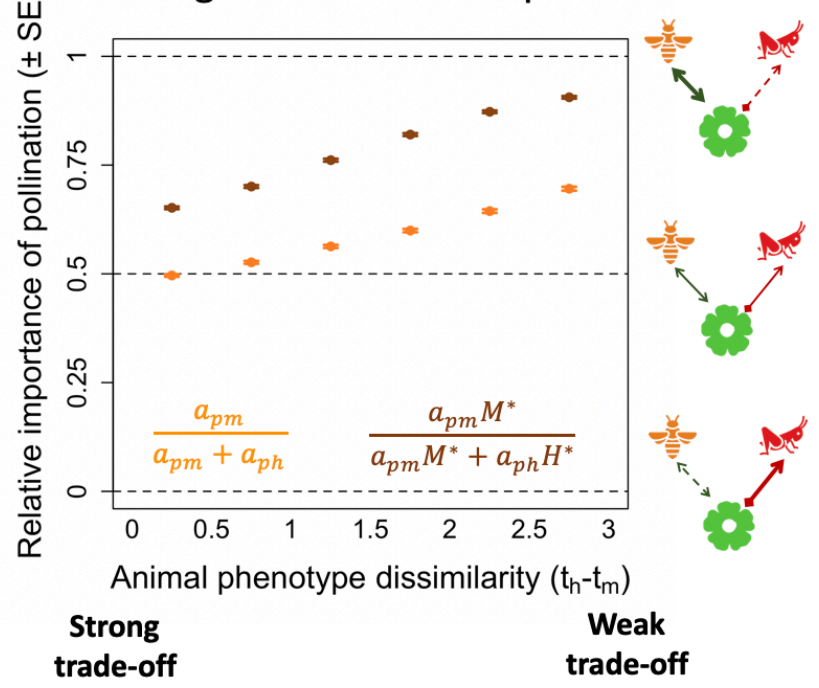

Fig. 3: Pollination to herbivory ratios constrain selection types and interaction patterns, for various tradeoff strengths. a. Distribution of pollination to herbivory ratio depending on selection type. The ratio is measured at the CSS for stabilizing selection, at the branching for disruptive selection, and at the repellor for runaway selection. The medians indicated correspond to absolute values (no log). The effect size of the KruskalWallis test $\left(p_{\text {value }}<2.10^{-16}\right)$ is also indicated. The stars indicate the significance level for the pairwise comparisons (Wilcoxon test): $* * *<10^{-4}$. b. Link between trade-off strength and selection type. The relative importance is measured as the proportion of the phenotypic space allowing stable coexistence over which a given type of selection is observed (see Fig. S2). c. Selected interaction pattern when coexistence is maintained. The cases where coexistence is maintained are under stabilizing selection (representing the vast majority of such cases), or in a dimorphic situation (extremely rare) resulting from disruptive selection. Coexistence is always disrupted under runaway selection. The results presented in this figure are from Monte-Carlo experiments (details in Appendix B.II). Interspecific parameter sets sampled: (a) 10000, (b) 1000. Ecological parameter set: $\left(r_{p}=\right.$ 
$10, r_{m}=-1, r_{h}=-4, c_{p}=0.6, c_{m}=0.5, c_{h}=0.4, e_{m}=0.2, e_{h}=0.3$ ). See Fig. S4 (Appendix C.II) for robustness.

Unraveling the influence of each plant-animal interaction parameter
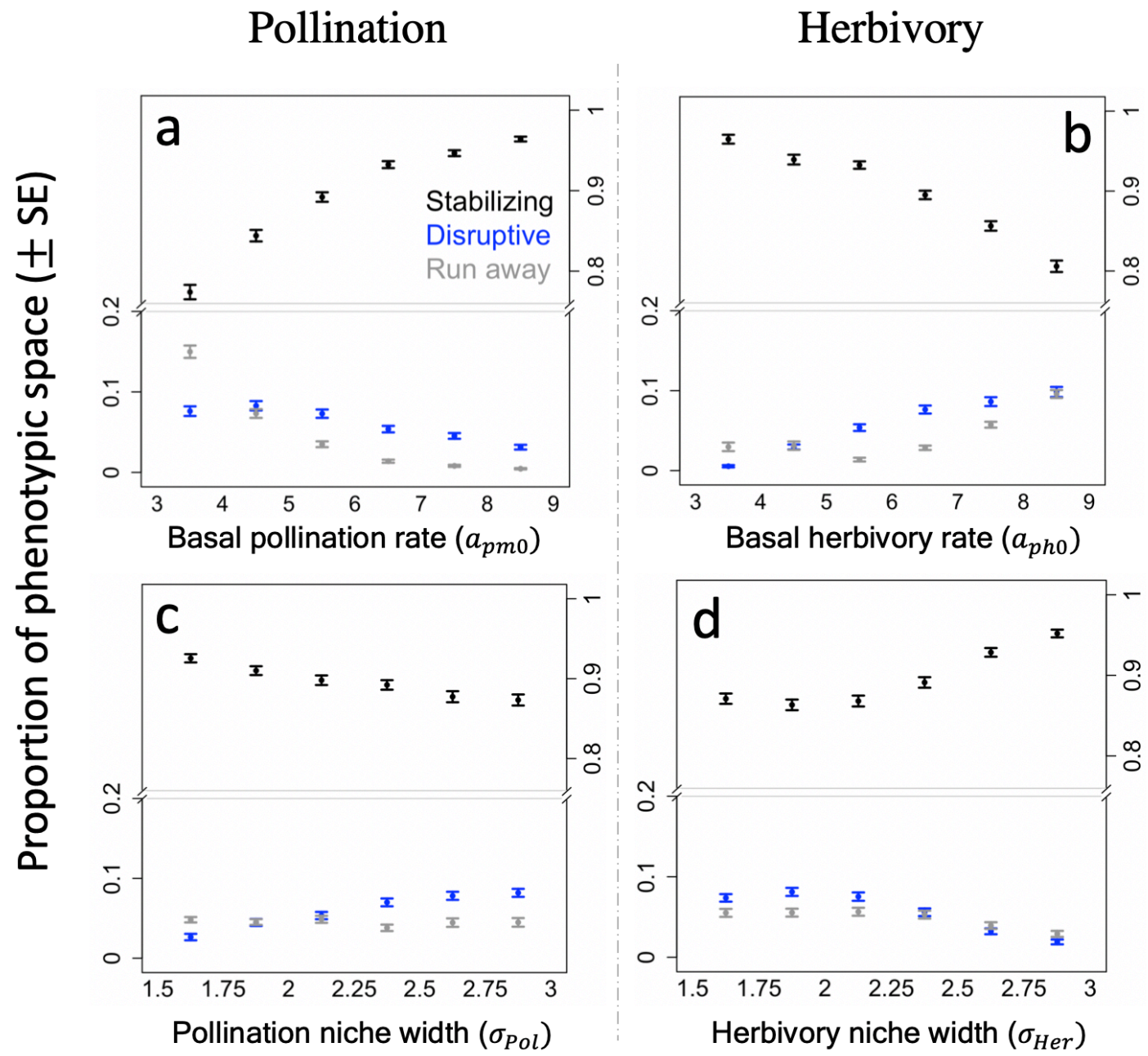

Fig. 4: Opposite effects of pollination and herbivory on selection. a. b. Variations in basal interaction rates.

c. d. Variations in niche widths. The results are from our fourth Monte-Carlo experiment (MC 4, 1000 sampled sets at each point, details in Appendix B.III). Ecological parameter set: $\left(r_{p}=10, r_{m}=-1, r_{h}=-4, c_{p}=\right.$ $0.6, c_{m}=0.5, c_{h}=0.4, e_{m}=0.2, e_{h}=0.3$ ). See Fig. S5 (Appendix D.I) for robustness.

Manipulating the pollination and herbivory niches further emphasizes the opposite influence of plant-animal interactions on eco-evolutionary dynamics (Fig. 4, 5 \& S6). The effects of basal rates and interaction niche widths on selection are obtained from a fourth Monte Carlo experiment (MC 4) in which a focal parameter is constrained within six successive interval ranges, exactly as done with trade-off intensity in MC2 (Fig. 3c). Increasing the basal 
rate of pollination $\left(a_{p m 0}\right)$ makes stabilizing selection more frequent at the expense of disruptive and runaway selection (Fig. 4a, e.g. Fig. 5a vs. 5b). On the contrary, higher herbivory rates $\left(a_{p h 0}\right)$ make disruptive and runaway selection more frequent at the expense of stabilizing selection (Fig. 4b, e.g. Fig. 5a vs. 5c). The same pattern is observed (Fig. 4d, e.g. Fig. S6b vs. S6d in appendix D.II) as the herbivory niche width $\left(\sigma_{H e r}\right)$ gets narrower. In contrast, a narrower pollination niche width $\left(\sigma_{P o l}\right)$ increases the prevalence of stabilizing selection (Fig. 4c, e.g. Fig. S6a vs. S6b). Variations are, however, less pronounced for niche widths $(\sigma)$ than for interaction rates $\left(a_{0}\right)$. Moreover, only in the case of interaction rates are these patterns robust to the variation of the ecological parameter set (Fig. S5, Appendix D.I).

The opposite impact of plant-animal interactions on selection implies opposite consequences for coexistence. Coexistence is favored by strong pollination $\left(a_{p m 0} \nearrow, \sigma_{P o l} \searrow\right)$ which provides stabilizing selection (Fig. 4a\&c, Fig. S5a). Coexistence is threatened by strong herbivory $\left(a_{p h 0} \nearrow, \sigma_{\text {Her }} \searrow\right.$ ) which often leads to runaway dynamics eventually destroying coexistence (Fig. 4b\&d, Fig. S5b). Very often (Fig 5\&S6), not only herbivores but both animal species are lost. Strong herbivory also displaces the CSS phenotypes closer to animal extinction thresholds (e.g. Fig. 5a vs. 5c, Fig. S6b vs. S6d), which implies the maintenance of a fragile coexistence. Given small animal densities, coexistence is indeed vulnerable to perturbations or stochasticity. Strong pollination, in contrast, displaces the CSS phenotypes away from extinction boundaries (e.g. Fig. 5c vs. 5d, S6a vs. S6b). Note, however, that strong pollination also favors the evolutionary emergence of unstable population dynamics, especially at weak trade-offs (unbounded growth in Fig. 5b\&d vs. 5a\&c).

Diversification of plant phenotypes through disruptive selection is fostered by strong herbivory (Fig. 4b\&d, Fig. S5b), and restricted by strong pollination (Fig. 4a\&c, Fig. S5a). Note that this is in line with balanced pollination to herbivory ratios $\left(\frac{a_{p m} M^{*}}{a_{p h} H^{*}}\right]_{t_{p}^{*}}$ Fig. 3a) because the ecological parameter set favors the density of pollinators over herbivores $\left(r_{m} \gg r_{h}\right)$. 
Dimorphism is rarely maintained and only at very strong trade-offs (i.e. $t_{h}-t_{m} \leq 0.5$ ). The maintenance of dimorphism nevertheless represents around $22 \%$ of the branchings at such strong trade-offs. Post-branching dynamics always lead to two morphs with qualitatively contrasting strategies, i.e. strong vs. weak plant-animal interactions (e.g. Fig. S7, appendix D.III).

\section{Small basal pollination rate$$
a_{p m 0}=5
$$
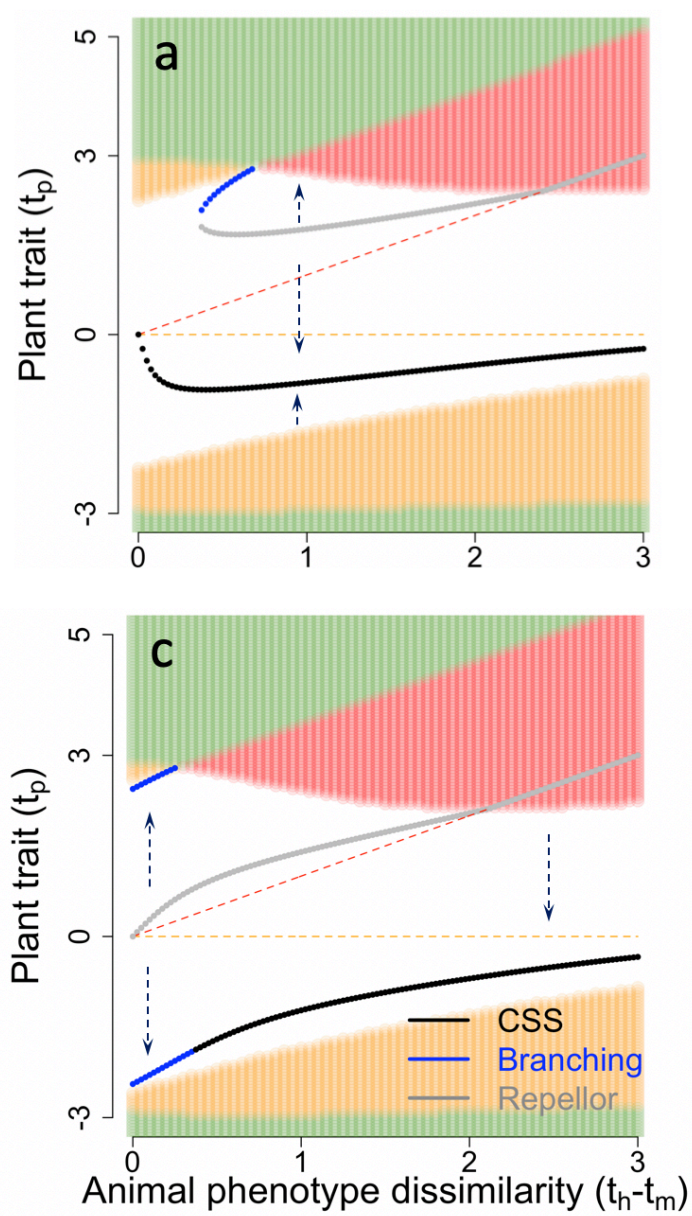
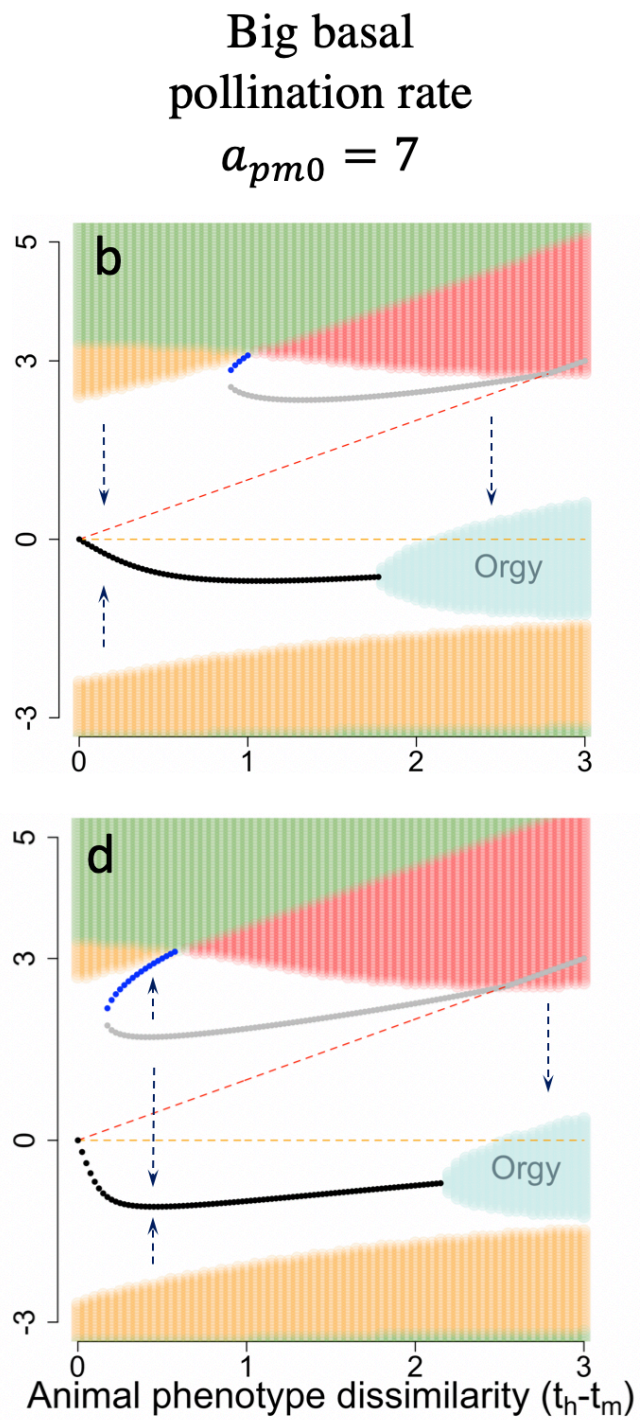

Fig. 5: Pollination vs. herbivory basal rates affect the community eco-evolutionary dynamics. Arrows indicate evolutionary trajectories and background colors indicate community composition (as in Fig. 2a): green for plants, orange for plant-pollinator, red for plant-herbivore and white for stable coexistence. Light blue indicates unstable population dynamics. Evolutionary singularities: CSS (black curve, convergent, noninvasible), Branching (blue curve, convergent, invasible) and repellor (grey curve, non-convergent, invasible). Arrows indicates Ecological parameter set: $\left(r_{p}=10, r_{m}=-1, r_{h}=-4, c_{p}=0.6, c_{m}=0.5, c_{h}=\right.$ $\left.0.4, e_{m}=0.2, e_{h}=0.3\right)$. Interaction niche widths: $\sigma_{\text {Pol }}=1.7, \sigma_{\text {Her }}=2$. 


\section{Discussion}

In this article, we investigate how the relative strength of pollination vs. herbivory drives plant-pollinator-herbivore eco-evolutionary dynamics when plant traits are affected by both interactions (ecological trade-off). Stronger pollination makes stabilizing selection more prevalent and facilitates coexistence. Stronger herbivory increases the prevalence of runaway selection, thereby disrupting coexistence. Importantly, disruptive selection can arise from the interplay of the two interactions, leading to plant diversification. Such a diversification requires strong or intermediate ecological trade-offs between attracting pollinators and escaping herbivores. Trade-off intensity therefore largely determines community eco-evolutionary dynamics, allowing pollination-dominated interaction patterns when weaker. Weaker trade-offs also lead to increasingly fragile plant-pollinator-herbivore coexistence.

In line with our predictions (Fig. 1A), we find that pollination fosters the convergence toward the pollinator phenotype - i.e. stabilizing selection - while herbivory favors the divergence from the herbivore phenotype - i.e. runaway selection. Such opposite selection pressures have notably been found acting on flower color (Irwin et al. 2003; Frey 2004), shape (Galen \& Cuba 2001) or display (Gómez 2005), nectar quantity (Adler \& Bronstein 2004) and flowering phenology (Brody 2008; Ehrlén \& Münzbergová 2009). Conceptually, all traits influencing the likelihood for a plant species to be discovered by its interacting species (persistence, relative abundance, size, fragrance, attractiveness...) - i.e. its "apparency" (Feeny 1976) - may experience ecological trade-offs. They are usually empirically uncovered by investigating the deviation of plant traits from optima favored by pollinators (Strauss \& Whittall 2006, see CSSs in Fig. 5). Using experimental evolution, Ramos \& Schiestl (2019) offer compelling evidence of such deviation. They show that plants that evolved in the presence of both interactions were less attractive to pollinators than those that evolved with pollinators, but 
still more attractive than plants that evolved with hand-pollination, with or without herbivores. The presence of pollinators thus selected toward plant-pollinator trait-matching, but the presence of herbivores reduced such a matching by adding a runway component to selection (equation (3)).

Short-generation plant species (e.g. Brassica rapa, Ramos \& Schiestl, 2019) offer a promising avenue to investigate real-time evolutionary trajectories of plant traits subject to the ecological trade-off between attracting pollinators and escaping herbivores. In line with the empirical evidence so far (Strauss et al. 2002; Strauss \& Irwin 2004), we find this trade-off to play a key role in the community eco-evolutionary dynamics. Using different animal species as selective agents (i.e. different interaction efficiencies) or varying animal densities would allow to experimentally manipulate trade-off intensities. Based on our results, experimental designs with high densities of a voracious herbivore should select plant phenotypes to which pollinators are maladapted, resulting in declining pollinator densities.

Our work underlines the selection of well-balanced interaction patterns at very strong trade-offs, while a bias toward stronger pollination is observed for weak trade-offs. In natural communities, however, the interaction pattern results from plant-animal coevolution rather than from the sole plant evolution (Thompson 2009), so that our result has to be taken cautiously. When animal morphology, preference or detection abilities evolve to match their resource (i.e. plant) phenotype, we expect such coevolution to lead to a strong ecological trade-off for the plant. Given our results, such situations would favor well-balanced interaction patterns and facilitate coexistence. We hypothesize that the commonness of shared preferences between pollinators and herbivores in natural communities (Strauss \& Whittall 2006) is partly driven by such coevolutionary dynamics. A more complete investigation of coevolutionary dynamics would be a natural extension of the present work. 
Similar animal preferences would presumably foster balanced patterns of pollination vs herbivory, which may favor coexistence (Mougi \& Kondoh 2012; Sauve et al. 2016a; Yacine \& Loeuille 2021). In the present model, we note that coexistence is fragile or destroyed for weak trade-offs (Fig. 3b\&c). Runaway dynamics are more frequent. In the vicinity of the pollinator phenotype, the plant phenotype increasingly stabilizes after the extinction of herbivores (i.e. Fig. 1B.1b), as indicated by the CSSs leaving the coexistence area (Fig. 3a). When stabilizing selection occurs before herbivore extinction, their low density makes them vulnerable to demographic stochasticity or Allee effects. Because weak ecological trade-offs are associated with fragile plant-pollinator-herbivore coexistence, they should be seldom encountered in natural communities. This is in line with the ubiquity of shared animal preferences for plant phenotype reported in the empirical literature (Adler \& Bronstein 2004; Strauss \& Whittall 2006).

Perhaps the most important result of our study is that disruptive selection can arise from the interplay of pollination and herbivory when ecological trade-offs are sufficiently strong. Polymorphism then yields two contrasted strategies: one that has many allies and enemies, while the other one has few interactions. Interestingly, such polymorphisms have been observed in empirical studies of ecological trade-offs. For instance, such trade-offs can lead to color polymorphism in both Raphanus sativus (Irwin et al. 2003) and Claytonia virginica (Frey 2004), and to floral display dimorphism in Primula farinosa (Ehrlén et al. 2002). Whether such mechanisms may act beyond polymorphisms and play important roles for speciation processes is an important question. Adler et al. (2012) used a phylogenetic analysis on 32 Nicotinia species and showed that variations in nicotine defenses among species are largely negatively correlated to pollinator reliance. These tobacco species are therefore either well-defended with few pollinators, or lowly defended and relying on pollination. The two contrasting plant 
strategies emerging here (strong vs. weak plant-animal interactions) also match certain observations made at the network scale that suggest that plant species with many pollinator partners also have more herbivores (Melián et al. 2009; Sauve et al. 2016b). Finally and in line with most previous theoretical works (Kopp \& Gavrilets 2006; Yoder \& Nuismer 2010; Maliet et al. 2020), our results emphasize the decisive role of herbivory in the emergence of disruptive selection (Fig. 4).

While dimorphism is often temporary here, this is largely due to how we modeled intraspecific competition. To focus on how trophic vs mutualistic interactions shape selection and its ecological consequences, we assumed constant competition rates. Considering similarity-dependent competition would likely favor the maintenance of dimorphism (Kisdi \& Geritz 1999; Doebeli \& Dieckmann 2000; Loeuille \& Hauzy 2018). We show that only 10\% of niche-overlap competition may suffice to maintain dimorphism (Fig. S8 in Appendix D.III). We expect similarity-dependent competition to be likely in natural communities as pollinators or herbivores using the same plants easily interfere (e.g. Campbell 1985), or because plant traits can be simultaneously involved in both competitive and plant-animal interactions (e.g. phenological traits, Schwinning \& Kelly 2013). Genetic correlations between such traits are also possible (e.g. plant size, Carmona et al. 2011). Reproductive isolation may then evolve as the morph that interacts weakly with animal species acquires new pollinators species (Baack et al. 2015), or evolves self-fertilization (Bodbyl Roels \& Kelly 2011). Disruptive selection from the interplay of pollination and herbivory can therefore be construed as a first step toward modeling the evolutionary emergence of plant-pollinator-herbivore networks, which would allow new insights into the eco-evolutionary processes supporting the diversity of natural communities. 


\section{Acknowledgements}

The authors would like to thank Dr. Sebastien Lion for his helpful feedback on the manuscript.

\section{References}

Adler, L.S. \& Bronstein, J.L. (2004). Attracting antagonists: Does floral nectar increase leaf herbivory? Ecology, $85,1519-1526$.

Adler, L.S., Seifert, M.G., Wink, M. \& Morse, G.E. (2012). Reliance on pollinators predicts defensive chemistry across tobacco species. Ecol. Lett., 15, 1140-1148.

Armbruster, W.S. (1997). Exaptations Link Evolution of Plant-Herbivore and Plant-Pollinator Interactions: A Phylogenetic Inquiry. Ecology, 78, 1661.

Baack, E., Melo, M.C., Rieseberg, L.H. \& Ortiz-Barrientos, D. (2015). The origins of reproductive isolation in plants. New Phytol., 207, 968-984.

Becerra, J.X., Noge, K. \& Venable, D.L. (2009). Macroevolutionary chemical escalation in an ancient plantherbivore arms race. Proc. Natl. Acad. Sci. U. S. A., 106, 18062-18066.

Bodbyl Roels, S.A. \& Kelly, J.K. (2011). Rapid evolution caused by pollinator loss in Mimulus guttatus. Evolution (N. Y)., 65, 2541-2552.

Bolnick, D.I. (2004). Can intraspecific competition drive disruptive selection? An experimental test in natural populations of sticklebacks. Evolution (N. Y)., 58, 608-618.

Brody, A.K. (2008). Effects of Pollinators , Herbivores, and Seed Predators on Flowering Phenology. Ecology, $78,1624-1631$.

Campbell, D.R. (1985). Pollinator Sharing and Seed Set of Stellaria pubera: Competition for Pollination. Ecology, 66, 544-553.

Carmona, D., Lajeunesse, M.J. \& Johnson, M.T.J. (2011). Plant traits that predict resistance to herbivores. Funct. Ecol., 25, 358-367.

Chomicki, G., Weber, M., Antonelli, A., Bascompte, J. \& Kiers, E.T. (2019). The Impact of Mutualisms on Species Richness. Trends Ecol. Evol., 34, 698-711.

Dawkins, R. \& Krebs, J.R. (1979). Arms races between and within species. Proc. R. Soc. B Biol. Sci., 205, 489511.

Dieckmann, U. \& Law, R. (1996). The dynamical theory of coevolution: a derivation from stochastic ecological processes. J. Math. Biol., 34, 579-612.

Doebeli, M. \& Dieckmann, U. (2000). Evolutionary Branching and Sympatric Speciation Caused by Different Types of Ecological Interactions. Am. Nat., 156, S77-S101.

Ehrlén, J., Käck, S. \& Ågren, J. (2002). Pollen limitation, seed predation and scape length in Primula farinosa. Oikos, 97, 45-51.

Ehrlén, J. \& Münzbergová, Z. (2009). Timing of flowering: Opposed selection on different fitness components and trait covariation. Am. Nat., 173, 819-830.

Ehrlich, P.R. \& Raven, P.H. (1964). Butterflies and Plants: A Study in Coevolution. Evolution (N. Y)., 18, 586. 
Eshel, I. (1983). Evolutionary and continuous stability. J. Theor. Biol., 103, 99-111.

Farrell, B.D., Dussourd, D.E. \& Mitter, C. (1991). Escalation of plant defense: do latex and resin canals spur plant diversification? Am. Nat., 138, 881-900.

Feeny, P. (1976). Chapter One. In: Plant apparency and chemical defense. pp. 1-40.

Fontaine, C., Guimarães Jr, P.R., Kéfi, S., Loeuille, N., Memmott, J., van der Putten, W.H., et al. (2011). The ecological and evolutionary implications of merging different types of networks. Ecol. Lett., 14, 11701181.

Frey, F.M. (2004). Opposing natural selection from herbivores and pathogens may maintain floral-color variation in Claytonia virginica (Portulacaceae). Evolution (N. Y)., 58, 2426-2437.

Galen, C. \& Cuba, J. (2001). Down the tube: Pollinators, predators, and the evolution of flower shape in the alpine skypilot, Polemonium viscosum. Evolution (N. Y)., 55, 1963-1971.

Geritz, S.A.H., Metz, J.A.J., Kisdi, É. \& Meszéna, G. (1997). Dynamics of adaptation and evolutionary branching. Phys. Rev. Lett., 78, 2024-2027.

Gómez, J.M. (2005). Non-additive effects of herbivores and pollinators on Erysimum mediohispanicum (Cruciferae) fitness. Oecologia, 143, 412-418.

Grant, V. (1949). Pollination Systems as Isolating Mechanisms in Angiosperms. Evolution (N. Y)., 3, 82-97.

Hernández-Hernández, T. \& Wiens, J.J. (2020). Why are there so many flowering plants? A multiscale analysis of plant diversification. Am. Nat., 195, 948-963.

Hodges, S.A. \& Arnold, M.L. (1995). Spurring plant diversification: Are floral nectar spurs a key innovation? Proc. R. Soc. B Biol. Sci., 262, 343-348.

Holt, R.D. (1977). Predation, apparent competition, and the structure of prey communities. Theor. Popul. Biol., 12, 197-229.

Irwin, R.E., Strauss, S.Y., Storz, S., Emerson, A. \& Guibert, G. (2003). The Role of Herbivores in the Maintenance of a Flower Color Polymorphism in Wild Radish. Ecology, 84, 1733-1743.

Kessler, A., Halitschke, R. \& Poveda, K. (2011). Herbivory-mediated pollinator limitation: Negative impacts of induced volatiles on plant-pollinator interactions. Ecology, 92, 1769-1780.

Kiester, A.R., Lande, R. \& Schemske, D.W. (1984). Models of coevolution and speciation in plants and their pollinators. Am. Nat., 124, 220-243.

Kisdi, É. \& Geritz, S.A.H. (1999). Adaptive dynamics in allele space: Evolution of genetic polymorphism by small mutations in a heterogeneous environment. Evolution (N. Y)., 53, 993-1008.

Kopp, M. \& Gavrilets, S. (2006). Multilocus genetics and the coevolution of quantitative traits. Evolution (N. Y)., 60, 1321-1336.

Loeuille, N. \& Hauzy, C. (2018). Multidimensionality of plant defenses and herbivore niches : implications for eco-evolutionary dynamics. J. Theor. Biol.

Maliet, O., Loeuille, N. \& Morlon, H. (2020). An individual-based model for the eco-evolutionary emergence of bipartite interaction networks. Ecol. Lett., 23, 1623-1634.

Mauricio, R. \& Rausher, M.D. (1997). Experimental manipulation of putative selective agents provides evidence for the role of natural enemies in the evolution of plant defense. Evolution (N. Y)., 51, 1435-1444.

Maynard Smith, J. \& Price, G.R. (1973). The logic of animal conflict. Nature, 246, 15-18.

Melián, C.J., Bascompte, J., Jordano, P. \& Křivan, V. (2009). Diversity in a complex ecological network with 
two interaction types. Oikos, 118, 122-130.

Metz, J.A.J., Nisbet, R.M. \& Geritz, S.A.H. (1992). How should we define "fitness" for general ecological scenarios? Trends Ecol. Evol., 7, 198-202.

Mougi, A. \& Kondoh, M. (2012). Diversity of interaction types and ecological community stability. Science, 337, 349-351.

Mougi, A. \& Kondoh, M. (2014). Instability of a hybrid module of antagonistic and mutualistic interactions. Popul. Ecol., 56, 257-263.

Ollerton, J., Winfree, R. \& Tarrant, S. (2011). How many flowering plants are pollinated by animals? Oikos, 120, 321-326.

Parachnowitsch, A.L. \& Kessler, A. (2010). Pollinators exert natural selection on flower size and floral display in Penstemon digitalis. New Phytol., 188, 393-402.

Ramos, S.E. \& Schiestl, F.P. (2019). Rapid plant evolution driven by the interaction of pollination and herbivory. Science, 364, 193-196.

Sargent, R.D. (2004). Floral symmetry affects speciation rates in angiosperms. Proc. R. Soc. B Biol. Sci., 271, 603-608

Sauve, A.M.C., Fontaine, C. \& Thébault, E. (2016a). Stability of a diamond-shaped module with multiple interaction types. Theor. Ecol., 9, 27-37.

Sauve, A.M.C., Thébault, E., Pocock, M.J.O. \& Fontaine, C. (2016b). How plants connect pollination and herbivory networks and their contribution to community stability. Ecology, 97, 908-917.

Schwinning, S. \& Kelly, C.K. (2013). Plant competition, temporal niches and implications for productivity and adaptability to climate change in water-limited environments. Funct. Ecol., 27, 886-897.

Strauss, S.Y. \& Irwin, R.E. (2004). Ecological and Evolutionary Consequences of Multispecies Plant-Animal Interactions. Annu. Rev. Ecol. Evol. Syst., 35, 435-466.

Strauss, S.Y., Irwin, R.E. \& Lambrix, V.M. (2004). Optimal defence theory and flower petal colour predict variation in the secondary chemistry of wild radish. J. Ecol., 92, 132-141.

Strauss, S.Y., Rudgers, J.A., Lau, J.A. \& Irwin, R.E. (2002). Direct and ecological costs of resistance to herbivory. Trends Ecol. Evol., 17, 278-285.

Strauss, S.Y. \& Whittall, J.B. (2006). Non-pollinator agents of selection on floral traits. In: Ecology and evolution of flowers (eds. Harder, L.D. \& Barrett, S.C.H.). Oxford University Press on Demand, New York, NY, USA, pp. 120-138.

Theis, N., Barber, N.A., Gillespie, S.D., Hazzard, R. V. \& Adler, L.S. (2014). Attracting mutualists and antagonists: Plant trait variation explains the distribution of specialist floral herbivores and pollinators on crops and wild gourds. Am. J. Bot., 101, 1314-1322.

Thompson, J.N. (2009). The coevolving web of life. Am. Nat., 173, 125-140.

Whittall, J.B. \& Hodges, S.A. (2007). Pollinator shifts drive increasingly long nectar spurs in columbine flowers. Nature, 447, 706-709.

Yacine, Y. \& Loeuille, N. (2021). Stable coexistence in plant-pollinator-herbivore communities requires balanced mutualistic vs antagonistic interactions. bioRxiv.

Yoder, J.B. \& Nuismer, S.L. (2010). When Does Coevolution Promote Diversification? Am. Nat., 176, 802-817. 